\title{
Adaptive Backoff Algorithm for Contention Window for Dense IEEE 802.11 WLANs
}

\author{
Ikram Syed and Byeong-hee Roh \\ Department of Computer Engineering, Ajou University, Suwon-si, Gyeonggi-do 16499, Republic of Korea \\ Correspondence should be addressed to Byeong-hee Roh; bhroh@ajou.ac.kr
}

Received 5 December 2015; Accepted 15 May 2016

Academic Editor: Pedro M. Ruiz

Copyright (C) 2016 I. Syed and B.-h. Roh. This is an open access article distributed under the Creative Commons Attribution License, which permits unrestricted use, distribution, and reproduction in any medium, provided the original work is properly cited.

The performance improvement in IEEE 802.11 WLANs in widely fluctuating network loads is a challenging task. To improve the performance in this saturated state, we develop an adaptive backoff algorithm that maximizes the system throughput, reduces the collision probability, and maintains a high fairness for the IEEE 802.11 DCF under dense network conditions. In this paper, we present two main advantages of the proposed ABA-CW algorithm. First, it estimates the number of active stations and then calculates an optimal contention window based on the active station number. Each station calculates the channel state probabilities by observing the channel for the total backoff period. Based on these channel states probabilities, each station can estimate the number of active stations in the network, after which it calculates the optimal CW utilizing the estimated active number of stations. To evaluate the proposed mechanism, we derive an analytical model to determine the network performance. From our results, the proposed ABA-CW mechanism achieved better system performance compared to fixed-CW (BEB, EIED, LILD, and SETL) and adaptive-CW (AMOCW, Idle Sense) mechanisms. The simulation results confirmed the outstanding performance of the proposed mechanism in that it led to a lower collision probability, higher throughput, and high fairness.

\section{Introduction}

Wireless local area networks (WLANs) are becoming the most popular and widely deployed networks worldwide. The reason for this success is its low deployment cost and the robust and flexible medium access control (MAC) protocol with coexistence capabilities. The IEEE 802.11-based WLANs have become the most popular wireless network standard, and the IEEE 802.11 standard [1] has two basic operation modes: distributed coordination function (DCF) and optional point coordination function (PCF). PCF is a centralized MAC protocol in which an access point (AP) coordinates with different stations by sending polling messages, with the aim of providing collision-free services. However, DCF is a contention-based access scheme and is based on the carrier sensing multiple access/collision avoidance (CSMA/CA) mechanism using the binary exponential backoff (BEB) algorithm.

The DCF is the fundamental MAC mechanism employed in the IEEE 802.11 [1] to enable random access to wireless channels. If a station wishes to transmit, it is required to listen for the channel status for an interval called the DCF interframe space (DIFS) interval. If the status is busy during the interval, the station defers its access to the channel for a backoff period, which is determined by $T_{\mathrm{bo}}=\operatorname{rand}(\mathrm{CW}) \times$ $T_{\text {slot }}$, where $T_{\text {bo }}$ and $T_{\text {slot }}$ denote the time for the backoff period and slot time, respectively. Further, $\operatorname{rand}(\cdot)$ is the random-number generation function. Thus, the performance of the IEEE 802.11 DCF depends mainly on the CW adjustment and backoff strategy [2-5].

The BEB algorithm is the main backoff scheme employed in the IEEE 802.11 DCF. However, in terms of the throughput and collision rate of the BEB algorithm, its performance decreases dramatically when the number of stations increases beyond a certain limit. Cali et al. [3] reported that the actual throughput is lower than the analytical throughput because the $\mathrm{CW}$ varies depending on the network status. An improper $\mathrm{CW}$ adjustment increases the collision probability such that the stations experience many collisions before reaching the optimal CW. Several studies have been carried out to calculate the optimal CW that will increase the throughput and decrease the collision probability. Next, we further classify 
the related works into two categories based on the CW adjustment schemes employed: the fixed-CW $\left(\mathrm{CW}_{\min }\right.$ and $\left.\mathrm{CW}_{\max }\right)$ mechanism and adaptive-CW adjustment mechanism.

In fixed-CW adjustment schemes, several algorithms have been proposed, such as exponential increase exponential decrease (EIED), exponential increase linear decrease (EILD), multiplicative increase multiplicative decrease (MIMD), and smart exponential threshold linear (SETL), to improve the network performance using different increment factors to adjust the CW size [6-14]. Unfortunately, none of these algorithms can cope with the significant fluctuations in the network state and consider the fixed initial CW, that is, $\mathrm{CW}_{\text {min }}$, regardless of the network conditions [15]. All of the abovementioned algorithms increase their CWs exponentially or linearly when a collision occurs and decrease the CW when a successful transmission occurs. The CW adjustment rule is usually based on the status of the last transmission attempt, which increases the collision probability and decreases the aggregated throughput when the number of stations increases. Thus, we deduce that both the fixed-CW and improper CW adjustment rules based on the states of the last transmission are the main causes for the reduced throughput, and they increase the collision probability of the network.

In adaptive-CW adjustment schemes, the CW adjusts dynamically according to the network conditions (number of active stations or network traffic load) regardless of the last transmission. The stations receive the channel-state information and coordinate with other stations to reduce the collisions and improve the network throughput. In [1620], a lot of local channel-state information (e.g., idle slot intervals, slot utilization, and collision rate) was utilized to characterize the dynamic network conditions (number of active stations or network traffic load). In [21], the authors utilized the idle slot interval to adjust the $\mathrm{CW}$ in order to obtain a higher throughput. However, these methods do not estimate the number of stations, which may lead to decreased fairness. Further, some studies [22-33] estimated the number of active stations and then adjusted the access parameters to realize further performance improvements. However, in DCF, it is difficult to estimate the number of stations because each station can enter or leave the network at any time.

In [25], the authors estimated the number of stations by considering the signs of activity originating from other stations. In [26], the authors observed the channel events to estimate the number of stations by using the channelevent probability, and they tuned the network to obtain high throughput with good fairness according to the number of stations. In [27], the authors proposed a mechanism to tune the CW size based on the number of active stations, and the number was estimated by observing the channel status. Peng et al. [28] proposed a method to determine the CW by optimizing the ratio between the idle period and the collision period in terms of the throughput. Bianchi et al. [7] proposed a method to determine the CW based on the number of active stations. The number of stations was estimated by the number of slot times that were observed to be busy because of transmissions by other stations. Cali et al. [15] proposed a method that estimates the number of stations using the number of empty slots and tunes the CW accordingly.

In [32], the authors proposed an Idle Sense algorithm, where each host observes the mean number of idle slots between two transmission attempts, and the hosts adjust their CW by comparing the estimate with a theoretically derived value. In [33], the authors reported an Idle Sense mechanism with three thresholds for estimating the number of active stations in the network, and they developed a linear $\mathrm{CW}$ adjustment rule based on that number. However, the aggregated throughput of these mechanisms can be further improved.

In this paper, we aim to develop a new adaptive and robust backoff algorithm that improves the performance in terms of maximizing the system throughput, reducing the collision probability, and ensuring good fairness for the DCF in an IEEE 802.11 WLAN under dense network conditions. In this paper, we present two main contributions of the proposed adaptive backoff algorithm for the contention window, named ABA-CW. First, it estimates the number of active stations and then calculates an optimal CW based on the number of active stations. Each station has to observe the channel for the total backoff period and calculate the channel-state probabilities for the backoff period. Based on these channel-state probabilities, each station can estimate the number of active stations on the network, after which it then calculates the optimal CW utilizing the estimated number of active stations. For the estimated number of active stations, the proposed scheme achieved an optimal $\mathrm{CW}$ value that gives a good network performance in terms of high throughput, low collision probability, better channel utilization, and good fairness as the number of stations on the network varies.

The remainder of the paper is organized as follows. In Section 2, we discuss the DCF mechanism and the motivation for the proposed work. In Section 3, we estimate the channel status and propose a simple but novel algorithm for estimating the number of active stations based on the channel-state probabilities. We also derived an optimal CW for the number of active stations. In Section 4, we present an analysis of the proposed scheme. The simulation results and discussion are presented in Section 5, and we conclude the paper in Section 6.

\section{DCF Optimization}

In this section, we briefly describe the DCF mechanism, as detailed in the IEEE 802.11 standard [1]. According to the DCF mechanism, when a station has a packet to transmit, it senses the channel for an idle period that is greater than a DIFS interval. If the channel remains idle, the station transmits the packet; otherwise, the transfer is deferred until the ongoing transmission is terminated. The station continues to monitor the channel until it is measured as idle for a DIFS interval, and it then generates a random backoff interval. Random backoff intervals are slotted, and stations are only allowed to transmit their packets at the beginning of each slot. The random backoff interval value is uniformly selected from the 


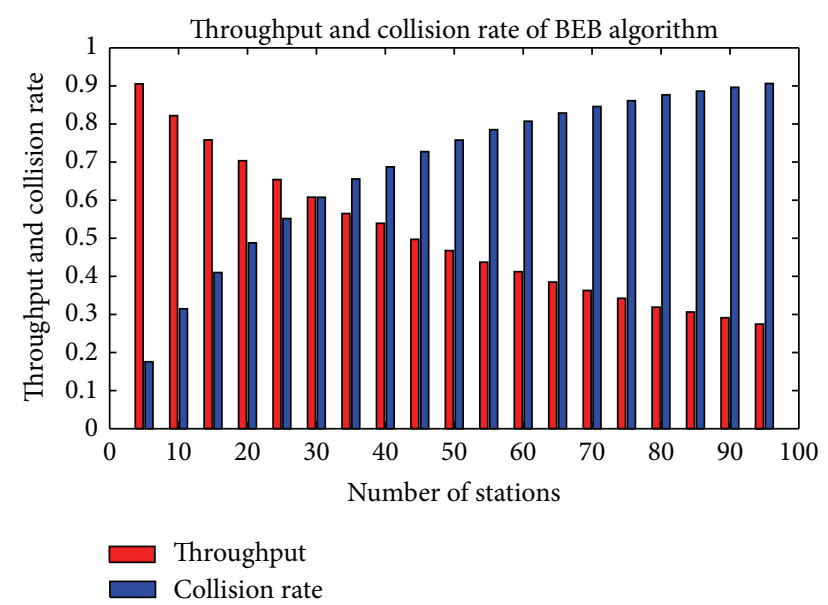

FIGURE 1: Number of stations versus throughput and collision rate.

range $[0, \mathrm{CW}-1]$ interval, where $\mathrm{CW}$ is the current $\mathrm{CW}$. The contention window size doubles with each collision, and this occurs when two or more stations transmit their packets in the same time slot. The backoff counter is frozen when activities are detected on the channel, and it is reactivated after it is sensed that the channel is again idle for more than a DIFS interval; the counter is decremented as long as it is sensed that the channel is idle. When the backoff timer reaches zero, the station attempts to transmit a packet at the beginning of the next time slot. If the packet is successfully received, the receiver sends an acknowledgment (ACK) after a short interframe space (SIFS), which is less than DIFS. If the packet transmission is unsuccessful as indicated by an ACK timeout, a retransmission is scheduled, and the CW increased by two with each unsuccessful transmission until it reaches its maximum value; that is, $\mathrm{CW}_{\max }=2^{m} \mathrm{CW}_{\min }$, where $m$ is the maximum backoff stage and its value could be 5 or 7 , depending on the maximum and minimum contention window size.

The main reason for the throughput degradation in DCF is the exponential backoff, which changes the CW based on the last transmission status. As shown in Figure 1, the BEB algorithm works well for a small number of stations. However, as the number of stations increases, the collision rate increases and the throughput significantly decreases. By making the $\mathrm{CW}$ dynamically adjust irrespective of the last transmission attempt, our proposed algorithm adjusts the CW based on the network conditions. It estimates the number of stations and then adjusts the CW based on the number of active stations in the network, which improves the network performance in terms of the throughput and collision probability.

\section{Adaptive Contention Window-Based Backoff Algorithm}

Our objective is to develop an adaptive backoff algorithm for IEEE 802.11 DCF wireless networks, which adjusts the CW dynamically with variations in the network load. As discussed above, fixed-CW backoff algorithms do not consider the network conditions when estimating the CW. As such, to realize successful transmissions, all of the algorithms reduce the $\mathrm{CW}$ exponentially or linearly, while increasing the $\mathrm{CW}$ for each collision that occurs. However, success/collision is not guaranteed when the network load becomes light or heavy, and this extreme variation in the $\mathrm{CW}$ affects the performance of the backoff algorithms. To overcome this problem, some recent research considered the channel-state parameters utilizing the network conditions in order to dynamically adjust the $\mathrm{CW}$, thus improving the throughput and fairness of the network. To adjust the CW adaptively in order to realize maximum throughput and to achieve good fairness, we derived an analytical model that utilizes the network conditions when calculating the optimal CW. The proposed $\mathrm{ABA}-\mathrm{CW}$ considers the network condition to adaptively vary the CW for both successful transmissions and collisions. The proposed algorithm has two main features: it estimates the number of active stations from the channel-state information, which is discussed in the next section. Then, it adjusts the $\mathrm{CW}$ according to the number of active stations on the network. The proposed algorithm adjusts the $\mathrm{CW}$ using the network load. It increases the $\mathrm{CW}$ when the network is dense and reduces it when the network load decreases. Thus, the proposed algorithm improves the network performance in terms of the throughput and collision probability. By finding the optimal CW based on the number of active stations, the proposed algorithm also provides an equal opportunity to all active stations, which also improves the fairness of the network.

3.1. Channel-State Estimation. In order to obtain actual channel status information, a station has to observe the channel for an entire backoff period. As shown in Figure 2, the channel status can be generally divided into three states, namely, the collision state, success state, and idle state. To calculate the channel-state probabilities, we consider $N$ slots in the total backoff period and $n$ stations, and the probability that one out of $n$ stations transmits data during a slot is given by

$$
P(X=l)=\left(\begin{array}{l}
n \\
l
\end{array}\right)\left(\frac{1}{N}\right)^{l}\left(1-\frac{1}{N}\right)^{n-l} .
$$

The number $l$ in a particular slot is called the occupancy number of that slot [34]. The expected number of slots, with the occupancy number $l$, is

$$
E(X=l)=N\left(\begin{array}{l}
n \\
l
\end{array}\right)\left(\frac{1}{N}\right)^{l}\left(1-\frac{1}{N}\right)^{n-l} .
$$

The probability of having an occupancy number 0 is given by

$$
P(X=0)=\left(1-\frac{1}{N}\right)^{n},
$$

and the probability of having an occupancy number 1 can be calculated by

$$
P(X=1)=\frac{n}{N} \cdot\left(1-\frac{1}{N}\right)^{n-1} .
$$




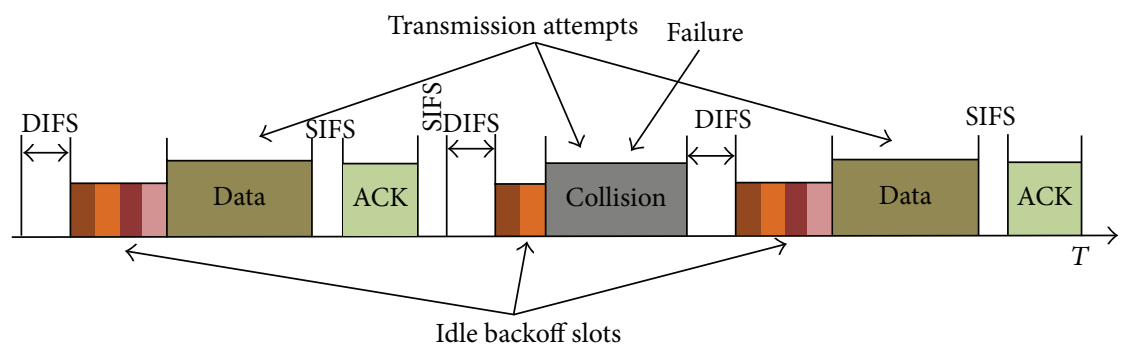

FIGURE 2: Basic DCF scheme.

A collision occurs when two or more stations transmit their data on the same slot at the same time, and the probability of having an occupancy number $\geq 2$ can be calculated using

$$
P(X \geq 2)=\left(\begin{array}{l}
n \\
l
\end{array}\right)\left(\frac{1}{N}\right)^{l}\left(1-\frac{1}{N}\right)^{n-l}, \quad \text { where } l \geq 2 .
$$

3.2. Contention Window Optimization. The throughput is a function of $P_{\text {succ }}, P_{\text {coll }}$, and $P_{\text {idle }}$, as shown in (6). The other parameters $\left(T_{\text {coll }}, T_{\text {succ }}\right.$, and $\left.T_{\text {idle }}\right)$ are constant with respect to the PHY parameters of the networks. We will discuss these parameters in detail in Section 4. $P_{\text {succ }}, P_{\text {coll }}$, and $P_{\text {idle }}$ are functions of CW and $n$. For a given $n$, there exists an optimal contention window, $\mathrm{CW}^{*}$, which achieves the maximum throughput. To find $\mathrm{CW}^{*}$, we take the partial derivative in (6) with respect to CW. To simplify the calculation, we take the partial derivative with respect to $\tau$ because $\tau$ is a function of CW. Using the quotient rule [35], the derivative of (6) is given as (7). Consider

$$
\begin{aligned}
& \theta=\frac{P_{\text {succ }}\left(T_{\text {hdr }}+T_{\text {pkt }}\right)}{\left(1-P_{\text {tr }}\right) T_{\text {idle }}+P_{\text {succ }} T_{\text {succ }}+P_{\text {coll }} T_{\text {coll }}}, \\
& \frac{d \theta}{d \tau}=\frac{\left(n \tau(1-\tau)^{n-1}\right) \cdot T_{\text {succ }}}{(1-\tau)^{n} \cdot\left(1-(1-\tau)^{n}\right) \cdot T_{\text {idle }}+\left(n \tau(1-\tau)^{n-1}\right) \cdot T_{\text {succ }}+\left(1-(1-\tau)^{n-1}\right) \cdot\left(1-(1-\tau)^{n}\right) \cdot T_{\text {coll }}}=0, \\
& {\left[(1-\tau)^{n} *\left\{1-(1-\tau)^{n}\right\} * T_{\text {idle }}+\left\{n \tau(1-\tau)^{n-1}\right\} * T_{\text {succ }}+\left\{1-(1-\tau)^{n-1}\right\} *\left\{1-(1-\tau)^{n}\right\} * T_{\text {coll }}\right] * n(1-\tau)^{n-2} *(1} \\
& \quad-n \tau) *\left(T_{\text {hdr }}+T_{\text {pkt }}\right)-\left\{n \tau(1-\tau)^{n-1}\right\} *\left(T_{\text {hdr }}+T_{\text {pkt }}\right) *\left[n\left\{2(1-\tau)^{n}-1\right\} *(1-\tau)^{n-1} * T_{\text {idle }}+n(1-\tau)^{n-2}\right. \\
& \left.\quad *(1-n \tau) * T_{\text {succ }}+(n-1) *(1-\tau)^{n-2} *\left\{1-(1-\tau)^{n}\right\} * T_{\text {coll }}\right]=0 .
\end{aligned}
$$

By solving (8), we obtain the following:

$$
\begin{aligned}
& n(1-\tau)^{2 n-2} \cdot T_{\text {idle }}+n^{2} \tau(1-\tau)^{2 n-2} \cdot T_{\text {coll }} \\
& \quad-n(1-\tau)^{2 n-2} \cdot T_{\text {coll }}+n(1-\tau)^{n-2} \cdot T_{\text {coll }} \\
& \quad-n^{2} \tau(1-\tau)^{n-2} \cdot T_{\text {coll }}=0 .
\end{aligned}
$$

By simplifying and then substituting the $\tau$ value into (9), where $\tau=(2 / \mathrm{CW}+1)$ [7], we obtain the relationship between $\mathrm{CW}^{*}$ and $n$ from the following:

$$
\mathrm{CW}^{*}=\left(\frac{\mathrm{CW}_{\min }}{2}\right) \times n-1,
$$

where $\mathrm{CW}_{\min }$ is the minimum contention window, while $\mathrm{CW}^{*}$ is the optimal contention window and $n$ is the number of stations in the network.

3.3. Accurate Estimation of Number of Active Stations. As shown in (10), $\mathrm{CW}^{*}$ depends on $\mathrm{CW}_{\text {min }}$ and the number of active stations on the network. Usually, this number is unknown and needs to be estimated. In this section, we find an accurate estimation of the number of active stations from the collision probability $P_{\text {coll }}$ and $\mathrm{CW}_{\text {min }}$ by replacing the $\tau$ value in (13) using (12) [7]. We obtain the estimated number of active stations $n_{\text {est }}$ as follows:

$$
n_{\mathrm{est}}=\frac{\log \left(1-\left(2 /\left(\mathrm{CW}_{\min }+1\right)\right)\right)+\log \left(1-P_{\text {coll }}\right)}{\log \left(1-\left(2 /\left(\mathrm{CW}_{\min }+1\right)\right)\right)},
$$

where $\mathrm{CW}_{\text {min }}$ depends on the PHY parameters of the network. The proposed ABA-CW scheme realized an almost constant $P_{\text {coll }}$ value of approximately 0.21 , which we used as a threshold collision probability, $\mathrm{Th}_{\mathrm{Coll}}$, in order to estimate the number of stations on the network. Detailed procedures for estimating the number of stations are summarized in Algorithm 1.

To estimate the number of active stations, we simulate the proposed algorithm on the network for $[0,15)$ s for 5 stations, $[15,30) \mathrm{s}$ for 10 stations, $[30,45) \mathrm{s}$ for 15 stations, and $[45,60) \mathrm{s}$ 
Step 1: Set $\mathrm{CW}_{\min }$, and set the threshold value for the collision probability $\mathrm{Th}_{\mathrm{Coll}}$.

Step 2: Calculate $P_{\text {Coll }}$ using (5).

Step 3: Compare $P_{\text {Coll }}$ with $\mathrm{Th}_{\text {Coll }}$.

while $\left(P_{\text {Coll }} \geq \mathrm{Th}_{\text {Coll }}\right)$

Estimate the number of active stations using (11).

Calculate $\mathrm{CW}_{\text {min }}$ obtained by (10) using $n_{\text {est }}$.

Update the $P_{\text {Coll }}$ value using (5).

End

Step 4: Estimate the number of active stations using the updated $P_{\mathrm{Coll}}$ and $\mathrm{CW}_{\min }$ obtained by (11).

Algorithm 1: Estimation of the number of active stations.

while (contention)

A station observes the channel for backoff period.

Calculate $P_{\text {Coll }}$ using (5).

Estimate $n_{\text {est }}$, following the procedure in Algorithm 1.

Calculate $\mathrm{CW}^{*}$ using (10).

Choose a random backoff value from the range $\left[0, \mathrm{CW}^{*}-1\right]$.

Decrement backoff timer by one when the channel is idle as DCF protocol.

End

Transmit a packet when the backoff timer expires.

Algorithm 2: Adaptive backoff algorithm of contention window.

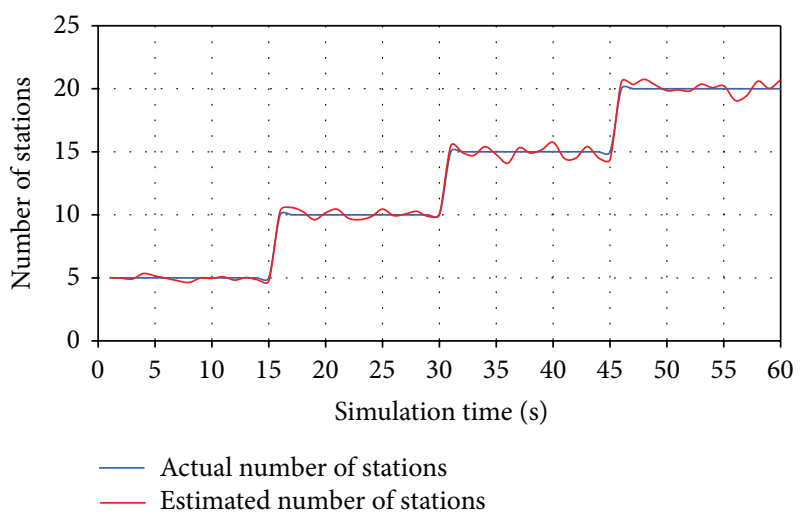

Figure 3: Estimated number of stations versus actual number of stations.

for 20 stations. As shown in Figure 3, the proposed scheme provides an accurate estimation of the number of stations on the network, which we subsequently use to calculate the optimal CW.

\subsection{Proposed Adaptive Backoff Algorithm for Contention} Window. Algorithm 2 shows a detailed operation process for ABA-CW. For all contentions, each station has to observe the channel for the total backoff period and calculate $P_{\text {coll }}$ using (5). Then, all of the stations estimate the number of active stations on the network using Algorithm 1 . Next, a station determines $\mathrm{CW}^{*}$ based on the number of active stations using (10). When a station receives a packet from a higher layer or another station, it randomly chooses an integer value using a uniform distribution over the interval [ 0 , $\mathrm{CW}^{*}-1$ ] to determine the backoff timer, and it then begins to perform the backoff procedure. The backoff procedure is the same as that of DCF. When the channel is idle, the backoff counter decreases by one for each slot time; however, when the channel is busy, the backoff counter is frozen. The packet transmits whenever the backoff timer expires.

\section{Performance Analysis of ABA-CW Algorithm}

4.1. Channel-State Probabilities. Channel-state probabilities have been extensively studied as they are among the critical performances metric employed to analyze IEEE 802.11 backoff algorithms. In this section, we calculate the channelstate probabilities for the proposed ABA-CW scheme; we represent the collision probability by $P_{\text {coll }}$, success probability by $P_{\text {succ }}$, and transmission probability by $P_{\text {tr }}$ for a saturated network under ideal channel conditions. For the simple mean-value analysis, the Bianchi model [7] is sufficient to obtain an accurate prediction of the channel-state probabilities for a saturated network under ideal channel conditions. We assume a fixed number of stations $n$ in saturated network conditions. In a saturated network, every station always has a packet to transmit. The Bianchi model assumes that each packet collides with constant and independent probability $P_{\text {coll }}$, which is independent of the channel state. Let $\tau$ be the probability that a station attempts to transmit in a randomly chosen slot time, which is given by

$$
\tau=\frac{2}{(\mathrm{CW}+1)} .
$$


To compute the collision probability $P_{\text {coll }}$ where at least one of the $(n-1)$ remaining stations transmits within a slot time, we use

$$
P_{\text {coll }}=1-(1-\tau)^{n-1}
$$

Using (12) and (13), we can find the values of $\tau$ and $P_{\text {coll }}$. Once we obtain the value of $\tau$, we can compute the transmission, idle, and success probabilities for a randomly chosen slot. Let $P_{\text {tr }}$ be the transmission probability, where there is at least one station attempting to transmit in the considered slot time. $P_{\mathrm{tr}}$ can be found as follows:

$$
P_{\mathrm{tr}}=1-(1-\tau)^{n}
$$

When there is no transmission in the channel, the idle probability $P_{\text {idle }}$ can be obtained by

$$
P_{\text {idle }}=1-P_{\text {tr }}
$$

and when only one station transmits the packet over the channel at the chosen slot time, the success probability, $P_{\text {succ }}$, is given by

$$
P_{\text {succ }}=\frac{\left\{n \cdot \tau \cdot(1-\tau)^{n-1}\right\}}{\left\{1-(1-\tau)^{n}\right\}} .
$$

4.2. Throughput Analysis. In order to compute the normalized system throughput, $\theta$, we need to compute the actual time lengths of the collision slot $T_{\text {coll }}$ and success slot $T_{\text {succ }}$. For the IEEE 802.11 DCF basic access mechanism, a successful transmission includes the time for the physical and MAC headers $\left(T_{\text {hdr }}\right)$, payload $\left(T_{\text {pkt }}\right)$, SIFS $\left(T_{\text {SIFS }}\right)$, DIFS $\left(T_{\text {DIFS }}\right)$, and an acknowledgment $\left(T_{\mathrm{ACK}}\right)$ from the intended receiver station, considering the propagation delay:

$$
\begin{gathered}
T_{\text {succ }}=T_{\mathrm{hdr}}+T_{\mathrm{pkt}}+T_{\mathrm{SIFS}}+T_{\mathrm{ACK}}+T_{\mathrm{DIFS}}+2 \cdot \delta, \\
T_{\text {coll }}=T_{\mathrm{hdr}}+T_{\mathrm{pkt}}^{*}+T_{\mathrm{SIFS}}+T_{\mathrm{ACK}}+T_{\mathrm{DIFS}}+2 \cdot \delta,
\end{gathered}
$$

where $\delta$ and $T_{\mathrm{pkt}}^{*}$ are the propagation delay and the longest packet payload involved in the collision, respectively. In this case, we assumed that all packets have the same size, and thus $T_{\text {pkt }}^{*}=T_{\text {pkt }}$.

Therefore, we can compute the throughput $\theta$ of the system as follows [7]:

$$
\theta=\frac{P_{\text {succ }}\left(T_{\mathrm{hdr}}+T_{\mathrm{pkt}}\right)}{\left(1-P_{\mathrm{tr}}\right) T_{\text {idle }}+P_{\text {succ }} T_{\text {succ }}+P_{\text {coll }} T_{\text {coll }}},
$$

where $T_{\text {idle }}$ is the slot time for a backoff window.

In order to achieve the maximum theoretical throughput limit, as seen in (18), we need to maximize the success probability $P_{\text {succ }}$, while reducing the collision probability $P_{\text {coll }}$ and idle backoff time of the DCF. For this reason, we need to select $\mathrm{CW}_{\text {opt }}$ to increase the throughput of the system while reducing the collision probability and idle backoff time.
4.3. Fairness Calculation. The fairness can indicate how fairly the resources are allocated among the stations. Fairness is an important problem, primarily in fixed-CW backoff algorithms. We use Jain's fairness index to calculate the fairness of the proposed ACW-BA algorithm [36]:

$$
\mathrm{FI}=\frac{\left(\sum_{i=1}^{n} \theta_{i}\right)^{2}}{n \sum_{i=1}^{n} \theta_{i}^{2}}
$$

Jain's fairness always lies between 0 and 1. A fairness index of 1 indicates a fair resource distribution among the stations, while 0 represents an unfair distribution among the stations.

4.4. Channel Utilization. The channel utilization is defined as the total effective transmission time $\left(T_{\text {trans }}\right)$ for all stations divided by the total simulation time $\left(T_{\text {slot }} * N_{\text {slot }}\right)$ as shown in Figure 11:

$$
\rho=\frac{T_{\text {trans }} * L_{\text {pkt }}}{T_{\text {slot }} * N_{\text {slot }}},
$$

where $T_{\text {trans }}, L_{\text {pkt }}, T_{\text {slot }}$, and $N_{\text {slot }}$ represent the transmission time, packet length, slot time, and the number of slots, respectively.

4.5. Delay Analysis. In this section, we briefly analyze the frame delay for CSMA/CA mechanism. In CSMA/CA, the frame delay is considered as the time between the frame generation and its successful reception. For the frame delay analysis, we considered [37] as reference model, where the backoff delay $D_{B}$ and the number of collisions are the main causes for the frame delay. The frame delay $D_{F}$ can be calculated by the following relation:

$$
D_{F}=P_{\text {coll }}\left(D_{B}+T_{\text {coll }}+T_{o}\right)+\left(D_{B}+T_{\text {succ }}\right),
$$

where a station has to wait $T_{o}$ time to sense the channel again after collision occurred.

\section{Performance Evaluation}

5.1. Performance Validation: Comparison with DCF Algorithm. For the performance validation, we compared the throughput, collision rate, utilization, and fairness of the proposed ABA-CW algorithm with the DCF algorithm, as shown in Table 1.

As shown in Table 1, the throughput, utilization, and fairness of the DCF algorithm decrease as the number of stations in the network increases, while the ABA-CW algorithm maintains a constant throughput, utilization, and fairness for a varying number of stations in the network. The collision rate of the DCF algorithm increases as the number of stations in the network increases, while the ABA$\mathrm{CW}$ achieved a collision rate of less than 0.25 collisions for every 100 stations on the network; this is less than the smallest collision rate of the DCF algorithm. Therefore, the proposed ABA-CW mechanism outperforms the DCF algorithm in terms of throughput, collision rate, utilization, and fairness.

To evaluate further the performance of the proposed ABA-CW scheme, we compared it to the fixed-CW mechanisms and adaptive-CW mechanisms. 
TABLE 1: Comparison of DCF and ABA-CW algorithms for $\mathrm{CW}=16$, data rate $=54 \mathrm{Mbps}$.

\begin{tabular}{lccccccccccc}
\hline Number of stations & $\mathbf{5}$ & $\mathbf{1 0}$ & $\mathbf{2 0}$ & $\mathbf{3 0}$ & $\mathbf{4 0}$ & $\mathbf{5 0}$ & $\mathbf{6 0}$ & $\mathbf{7 0}$ & $\mathbf{8 0}$ & $\mathbf{9 0}$ & $\mathbf{9 5}$ \\
BEB throughput & 39.78 & 37.92 & 35.37 & 33.62 & 32.30 & 31.24 & 30.30 & 29.55 & 28.82 & 28.12 & 27.82 \\
ABA-CW throughput & 39.00 & 38.46 & 38.22 & 38.09 & 38.08 & 37.95 & 37.97 & 37.90 & 37.90 & 37.87 & 37.87 \\
BEB collision rate & 0.27 & 0.38 & 0.47 & 0.53 & 0.57 & 0.60 & 0.62 & 0.64 & 0.66 & 0.67 & 0.68 \\
ABA-CW collision rate & 0.18 & 0.20 & 0.21 & 0.22 & 0.22 & 0.22 & 0.23 & 0.23 & 0.23 & 0.24 & 0.24 \\
BEB utilization & 0.69 & 0.66 & 0.61 & 0.58 & 0.56 & 0.54 & 0.53 & 0.51 & 0.50 & 0.49 & 0.48 \\
ABA-CW utilization & 0.68 & 0.67 & 0.66 & 0.66 & 0.66 & 0.66 & 0.66 & 0.66 & 0.66 & 0.66 & 0.66 \\
BEB fairness & 0.99 & 0.97 & 0.94 & 0.92 & 0.89 & 0.88 & 0.86 & 0.85 & 0.83 & 0.82 & 0.82 \\
ABA-CW fairness & 0.99 & 0.99 & 0.99 & 0.99 & 0.99 & 0.99 & 0.99 & 0.99 & 0.99 & 0.99 & 0.99 \\
\hline
\end{tabular}

TABLE 2: Simulation parameters for IEEE 802.11g.

\begin{tabular}{llc}
\hline Symbol & Description & Value \\
\hline$L_{\text {DATA }}$ & Size of payload (bits) & 8,184 \\
$L_{\text {MAC }}$ & Size of MAC header (bits) & 272 \\
$L_{\text {PHY }}$ & Size of PHY header (bits) & 128 \\
$L_{\text {ACK }}$ & Size of ACK (bits) & 112 \\
$R$ & Data rate (Mbits $/$ s) & 24 \\
$T_{\text {slot }}$ & Length of slot time $(\mu$ s) & 9 \\
$T_{\text {SIFS }}$ & Length of short interframe space $(\mu \mathrm{s})$ & 10 \\
$T_{\text {DIFS }}$ & Length of DCF interframe space $(\mu \mathrm{s})$ & 28 \\
\hline
\end{tabular}

5.2. Comparison with Fixed-CW Mechanisms. In order to evaluate the performance of ABA-CW, we first compared the proposed algorithm with the fixed-CW mechanisms BEB [1], EIED [9], LILD, and SETL [14] as described in the related works. For the simulation, we consider an IEEE 802.11g [1] saturated network scenario comprised of $n$ stations that always have data packets in their queues to transmit. We developed the simulation model using MATLAB. Table 2 summarizes the simulation parameters for the proposed ABA-CW, BEB, EIED, LILD, and SETL. Considering IEEE $802.11 \mathrm{~g}$ as the physical medium, we set $L_{\mathrm{PHY}}, T_{\text {slot }}, T_{\mathrm{SIFS}}$, and $T_{\text {DIFS }}$ to 128 bits, $9 \mu \mathrm{s}, 10 \mu \mathrm{s}$, and $28 \mu \mathrm{s}$, respectively. In addition, we set the payload, MAC header, and acknowledgment sizes to 8,184 bits, 272 bits, and 112 bits, respectively. We set the channel rate to $24 \mathrm{Mbits} / \mathrm{s}$.

To evaluate the performance of ABA-CW, we compared it with the BEB, EIED, LILD, and SETL backoff algorithms. We performed the simulation and evaluation while varying the number of stations.

Figure 4 illustrates the performance comparison of the ABA-CW scheme with the fixed-CW mechanisms with respect to the collision probability. The horizontal axis represents the number of stations, while the vertical axis is the collision probability. As illustrated in Figure 4, the collision probability of the fixed-CW schemes increases as the number of stations in the network increases, while the proposed ABACW algorithm gives an almost constant collision probability regardless of the number of stations in the network. The four algorithms (fixed-CW mechanisms) only increase or decrease their CWs with their rules depending on whether the transmitted packets collided. These result in changes in the collision probability although the number of stations is

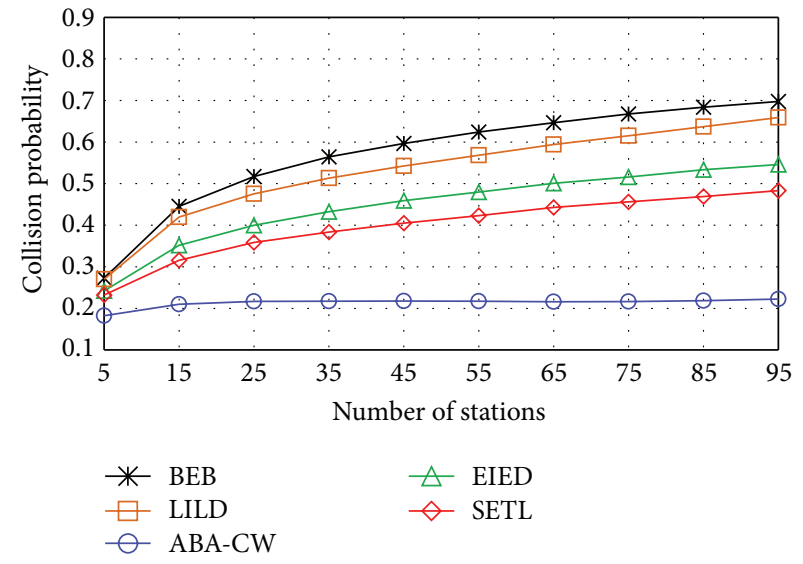

FIgURE 4: Collision probability versus the number of stations.

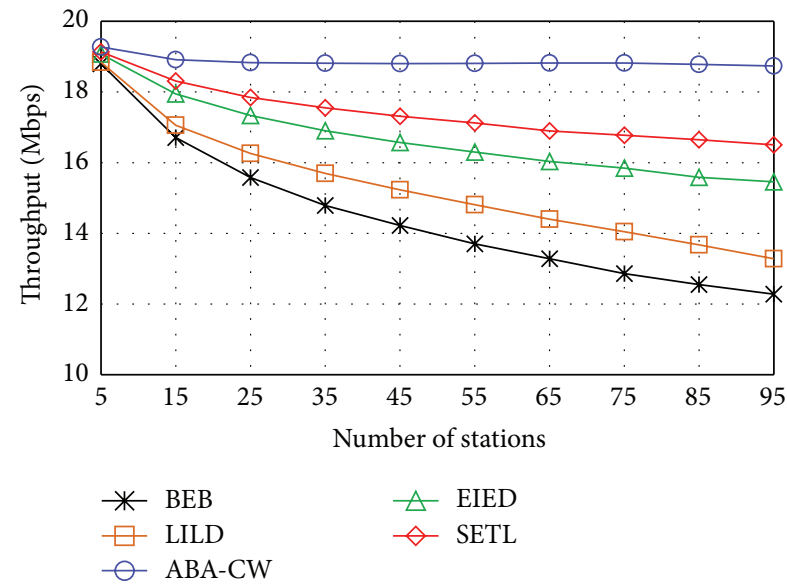

FIgURE 5: Throughput versus the number of stations.

fixed. However, the proposed scheme adjusts the CW based on the number of stations in the network.

Figure 5 shows a comparison of the proposed ABA-CW scheme with the existing fixed-CW schemes in terms of throughput. The horizontal axis represents the number of stations, while the vertical axis represents the throughput of the backoff schemes. The throughput of the fixed-CW schemes decreases as the number of stations increases, while 


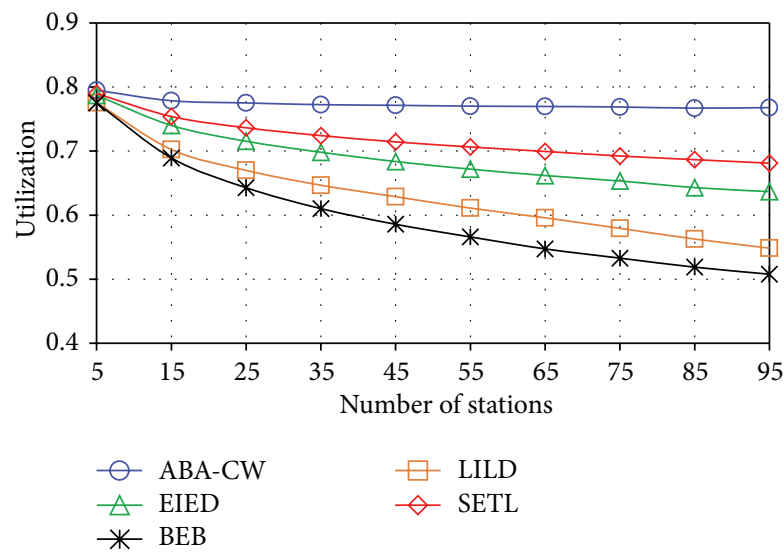

FIGURE 6: Channel utilization versus the number of stations.

the ABA-CW scheme achieves an almost constant throughput for different numbers of stations. Therefore, the proposed ABA-CW scheme performs better and achieves an almost constant throughput for the reasons explained for Figure 4. The probability of realizing stable collisions is directly related to a constant and high throughput.

The channel utilization is an important performance matric in the performance analysis of backoff algorithms. Most of the fixed-CW schemes do not utilize the channel in optimum way, which leads to a lower throughput for the fixed-CW schemes. Figure 6 shows a comparison of the ABA$\mathrm{CW}$ scheme with the fixed-CW schemes in terms of the channel utilization. The horizontal axis represents the number of stations, while the vertical axis represents the utilization. As shown in Figure 6, the channel utilization of the fixed$\mathrm{CW}$ schemes decreases as the number of stations in the network increases, while the proposed ABA-CW utilization is not affected by the number of stations in the network. We achieve almost constant utilization as the number of stations varies. The reason for this is that the proposed ABA-CW scheme considered the number of stations when calculating the optimal CW, which reduced the idle backoff time and improved the channel utilization for the proposed scheme.

Figure 7 shows a comparison of the ABA-CW and fixedCW schemes in term of fairness, which ranges from 0 to 1. As stated above, a fairness index of 1 indicates a fair resource distribution among the stations, while 0 represents an unfair distribution among the stations. The fairness index of the proposed ABA-CW scheme is almost 1 regardless of the number of stations, while for all of the fixed-CW schemes, with the exception of LILD, the fairness index decreases as the number of stations increases.

Figure 8 shows the frame delay analysis of the proposed ABA-CW in comparison with the fixed-CW (BEB, EIED, LILD, and SETL) schemes. The horizontal axis is the number of stations while vertical axis is the frame delay in $\mu$ s. As we observed, the proposed ABA-CW scheme achieved less frame delay in comparison with fixed-CW scheme. The main reason for the small frame delay is the lower collision rate and small backoff delay of the proposed scheme in comparison with the existing fixed-CW schemes.
TABLE 3: Simulation parameters for adaptive-CW mechanisms.

\begin{tabular}{llc}
\hline Symbol & Description & Value \\
\hline$L_{\text {DATA }}$ & Size of payload (bits) & 8,184 \\
$L_{\text {MAC }}$ & Size of MAC header (bits) & 272 \\
$L_{\text {PHY }}$ & Size of PHY header (bits) & 128 \\
$L_{\text {ACK }}$ & Size of ACK (bits) & 112 \\
$R$ & Data rate (Mbits $/ \mathrm{s})$ & 54 \\
$T_{\text {slot }}$ & Length of slot time $(\mu \mathrm{s})$ & 9 \\
$T_{\text {SIFS }}$ & Length of short interframe space $(\mu \mathrm{s})$ & 10 \\
$T_{\text {DIFS }}$ & Length of DCF interframe space $(\mu \mathrm{s})$ & 28 \\
\hline
\end{tabular}

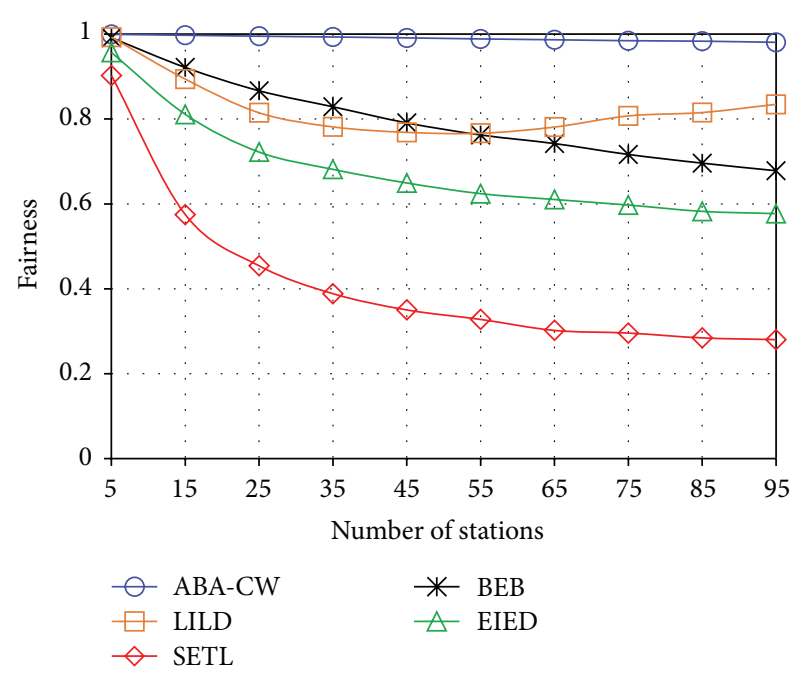

FIGURE 7: Fairness versus the number of stations.

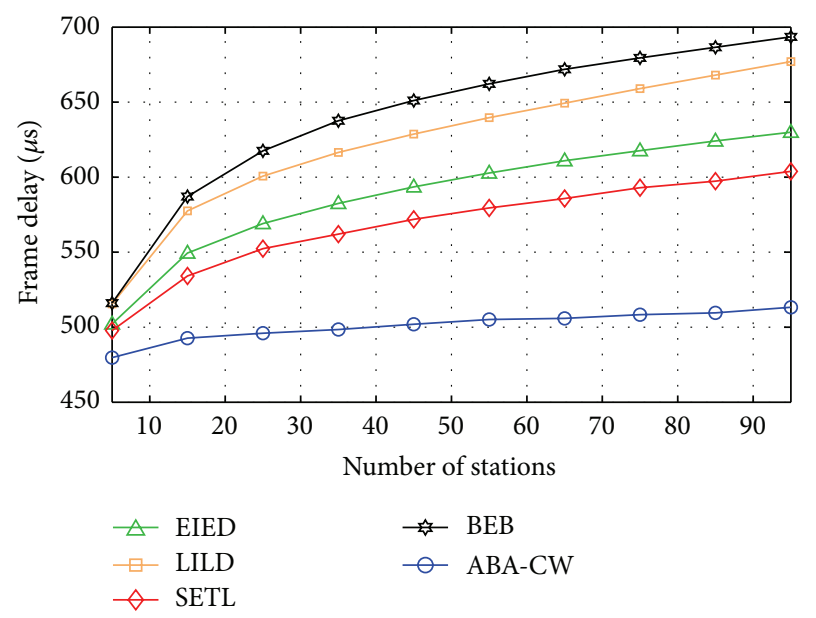

FIGURE 8: Frame delay analysis of the ABA-CW scheme versus fixedCW schemes.

5.3. Comparison with Adaptive-CW Mechanisms. To evaluate further the performance of the proposed ABA-CW scheme, we compared the proposed algorithm with the adaptive-CW mechanisms (Idle Sense [32], AMOCW [33]). Table 3 shows a summary of the simulation parameters for the proposed ABA-CW, AMOCW, and Idle Sense schemes. 


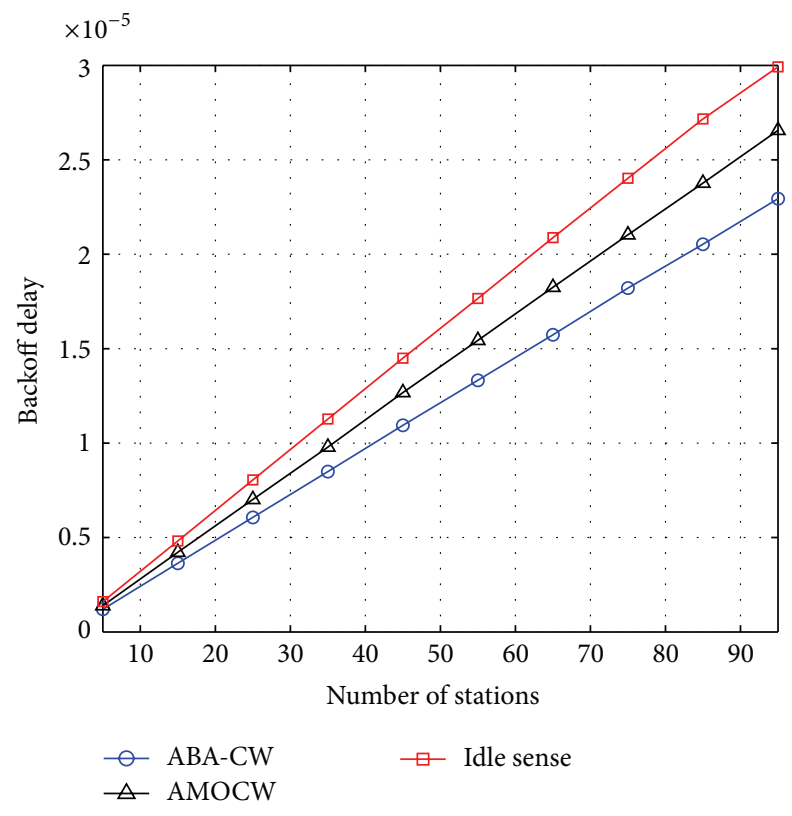

FIGURE 9: Backoff time versus the number of stations.

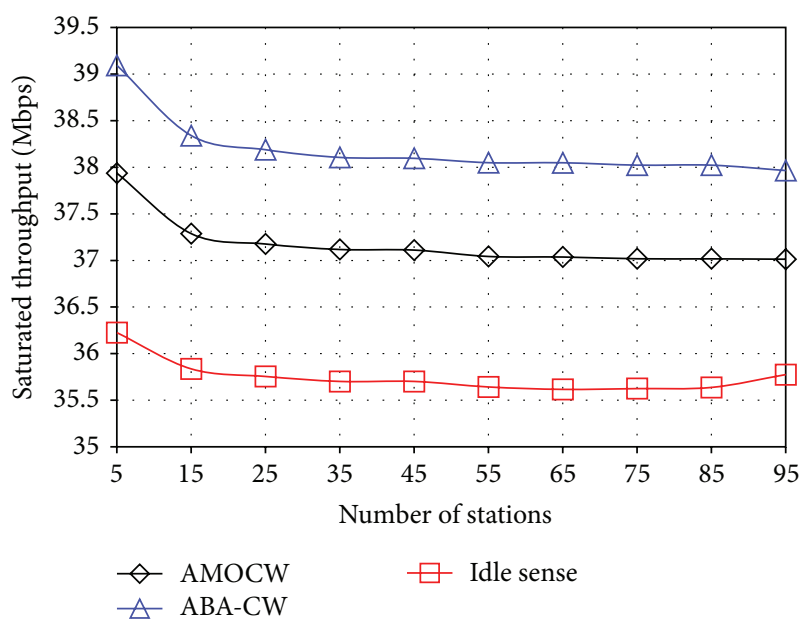

FIgURE 10: Throughput versus the number of stations.

In high data networks, the large idle backoff time degrades the performance of the network. As shows in Figure 8, the proposed ABA-CW spends less time in backoff procedure compared with the AMOCW and Idle Sense schemes, and this is one reason for the better system performance of the proposed scheme compared with the AMOCW and Idle Sense adaptive schemes.

Figure 9 shows the comparison of the proposed ABACW scheme with the AMOCW and Idle Sense adaptive$\mathrm{CW}$ schemes, and the proposed scheme achieves a higher throughput as the number of stations varies compared with the AMOCW and Idle Sense schemes. In all three schemes, the throughput is almost immune to changes in the number of stations in the network.

Figure 10 shows the channel utilization of the three adaptive schemes as the number of stations in the network varies.

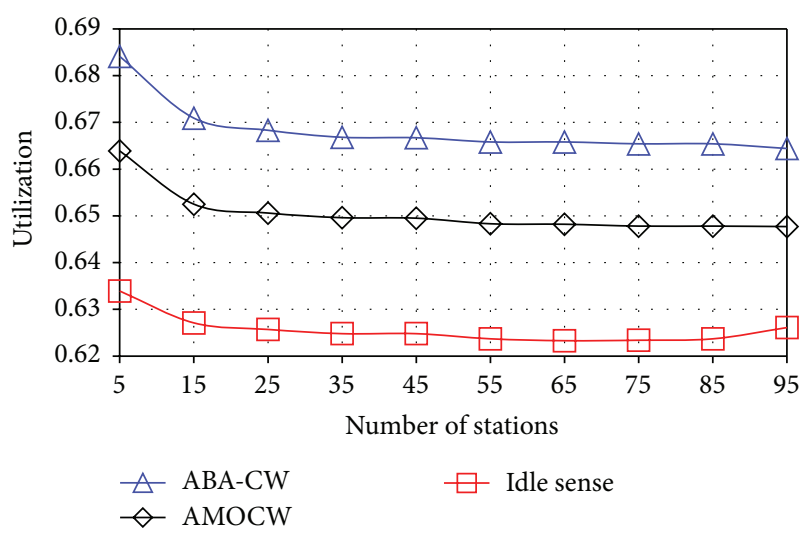

FIGURE 11: Channel utilization versus the number of stations.

The ABA-CW scheme maintains higher utilization compared to the AMOCW and Idle Sense adaptive mechanisms.

\section{Conclusion}

In this paper, we studied the problem of performance degradation in WLANs and proposed a simple but effective algorithm for improving the performance. In the proposed scheme, we aim to improve the saturated throughput, while maintaining a high fairness. To achieve this objective, we proposed an adaptive backoff algorithm for contention windows for IEEE 802.11 DCF. The proposed algorithm observes the channel status for a backoff period and uses the status to calculate the channel-state probabilities. Based on the channel-state probabilities, the proposed algorithm estimates the number of stations in the network. The algorithm then utilizes the number of stations to calculate the optimal CW. We further present an analytical model to analyze the properties of the ABA-CW mechanism, namely, channelstate probabilities, aggregated throughput, and fairness. The simulation results confirmed the outstanding performance of the proposed mechanism in terms of throughput, collision rate, utilization, and fairness.

\section{Competing Interests}

The authors declare that there are no competing interests regarding the publication of this paper.

\section{Acknowledgments}

This work was supported by the National Research Foundation of Korea (NRF) grant funded by the Korean Government (MSIP) (no. NRF-2015R1A2A2A01005577).

\section{References}

[1] IEEE Standard, "Wireless LAN Medium Access Control (MAC) and Physical Layer (PHY) specifications," IEEE Standard 802.111999, 1999.

[2] G. Bianchi, "Performance analysis of the IEEE 802.11 distributed coordination function," IEEE Journal on Selected Areas in Communications, vol. 18, no. 3, pp. 535-547, 2000. 
[3] F. Cali, M. Conti, and E. Gregori, "Dynamic tuning of the IEEE 802.11 protocol to achieve a theoretical throughput limit," IEEE/ACM Transactions on Networking, vol. 8, no. 6, pp. 785799, 2000.

[4] A. Kumar, E. Altman, D. Miorandi, and M. Goyal, "New insights from a fixed point analysis of single cell IEEE 802.11 WLANs," in Proceedings of the IEEE 24th Annual Joint Conference of the IEEE Computer and Communications Societies (IEEE INFOCOM '05), vol. 3, pp. 1550-1561, Miami, Fla, USA, March 2005.

[5] K. Medepalli and F. A. Tobagi, “Towards performance modeling of IEEE 802.11 based wireless networks: a unified framework and its applications," in Proceedings of the 25th IEEE International Conference on Computer Communications (INFOCOM '06), April 2006.

[6] P. Chatzimisios, V. Vitsas, and A. C. Boucouvalas, "Throughput and delay analysis of IEEE 802.11 protocol," in Proceedings of the IEEE 5th International Workshop on Networked Appliances, pp. 168-174, Liverpool, UK, October 2002.

[7] G. Bianchi, L. Fratta, and M. Oliveri, "Performance evaluation and enhancement of the CSMA/CA MAC protocol for 802.11 wireless LANs," in Proceedings of the 17th IEEE International Symposium on Personal, Indoor, and Mobile Radio Communication (PIMRC '96), Taipei, Taiwan, October 1996.

[8] N. I. Sarkar, "Improving WLAN performance by modifying an IEEE 802.11 protocol," International Journal of Wireless Networks and Broadband Technologies, vol. 1, pp. 15-31, 2011.

[9] N. O. Song, B. J. Kwak, J. Song, and L. E. Miller, "Enhancement of IEEE 802.11 distributed coordination function with exponential increase exponential decrease backoff algorithm," in Proceedings of the 57th IEEE Semiannual Vehicular Technology Conference (VTC '03), vol. 4, pp. 2775-2778, Jeju, South Korea, April 2003.

[10] V. Bharghavan, A. Demers, S. Shenker, and L. Zhang, "MACAW: a media access protocol for wireless LAN," in Proceedings of the ACM Conference on Communications Architectures, Protocols and Applications (SIGCOMM '94), vol. 24, no. 4, pp. 212-225, London, UK, October 1994.

[11] J. Deng, P. K. Varshney, and Z. J. Haas, "A new backoff algorithm for the IEEE 802.11 distributed coordination function," in Proceedings of the Communication Networks and Distributed Systems Modeling and Simulation Conference, San Diego, Calif, USA, 2004.

[12] Y. He, R. Yuan, J. Sun, and W. Gong, "Semi-random backoff: towards resource reservation for channel access in wireless LANs," in Proceedings of the 17th IEEE International Conference on Network Protocols (ICNP'09), pp. 21-30, IEEE, Princeton, NJ, USA, October 2009.

[13] Q. Nasir and M. Albalt, "History based adaptive backoff (HBAB) IEEE 802.11 MAC protocol," in Proceedings of the 6th Annual Communication Networks and Services Research Conference (CNSR '08), pp. 533-538, IEEE, Halifax, Canada, May 2008.

[14] C.-H. Ke, C.-C. Wei, K. W. Lin, and J.-W. Ding, "A smart exponential-threshold-linear backoff mechanism for IEEE 802.11 WLANs," International Journal of Communication Systems, vol. 24, no. 8, pp. 1033-1048, 2011.

[15] F. Cali, M. Conti, and E. Gregori, "IEEE 802.11 protocol: design and performance evaluation of an adaptive backoff mechanism," IEEE Journal on Selected Areas in Communications, vol. 18, no. 9, pp. 1774-1786, 2000.

[16] Y. Kwon, Y. Fang, and H. Latchman, "Design of MAC protocols with fast collision resolution for wireless local area networks,"
IEEE Transactions on Wireless Communications, vol. 3, no. 3, pp. 793-807, 2004.

[17] Y. Kwon, Y. Fang, and H. Latchman, "A novel MAC protocol with fast collision resolution for wireless LANs," in Proceedings of the 22nd Annual Joint Conference on the IEEE Computer and Communications Societies (INFOCOM '03), pp. 853-862, April 2003.

[18] L. Bononi, M. Conti, and E. Gregori, "Runtime optimization of IEEE 802.11 Wireless LANs performance," IEEE Transactions on Parallel and Distributed Systems, vol. 15, no. 1, pp. 66-80, 2004.

[19] L. Romdhani, Q. Ni, and T. Turletti, "Adaptive EDCF: enhanced service differentiation for IEEE 802.11 wireless ad-hoc networks," in Proceedings of the IEEE Wireless Communications and Networking Conference: The Dawn of Pervasive Communication (WCNC '03), pp. 1373-1378, IEEE, New Orleans, La, USA, March 2003.

[20] L. Chen, S. H. Low, and J. C. Doyle, "Random access game and medium access control design," IEEE/ACM Transactions on Networking, vol. 18, no. 4, pp. 1303-1316, 2010.

[21] X. Tian, X. Chen, T. Ideguchi, and Y. Fang, "Improving throughput and fairness in WLANs through dynamically optimizing backoff," IEICE Transactions on Communications, vol. E88-B, no. 11, pp. 4328-4338, 2005.

[22] T. Vercauteren, A. L. Toledo, and X. Wang, "Batch and sequential Bayesian estimators of the number of active terminals in an IEEE 802.11 network," IEEE Transactions on Signal Processing, vol. 55, no. 2, pp. 437-450, 2007.

[23] G. Bianchi and I. Tinnirello, "Kalman filter estimation of the number of competing terminals in an IEEE 802.11 network," in Proceedings of the 22nd Annual Joint Conference on the IEEE Computer and Communications Societies (INFOCOM '03), vol. 2, pp. 844-852, April 2003.

[24] S.-W. Kang, J.-R. Cha, and J.-H. Kim, "A novel estimation-based backoff algorithm in the IEEE 802.11 based wireless network," in Proceedings of the 7th IEEE Consumer Communications and Networking Conference (CCNC '10), pp. 1-5, IEEE, Las Vegas, Nev, USA, January 2010.

[25] M. Ibrahim and S. Alouf, "Design and analysis of an adaptive backoff algorithm for IEEE 802.11 DCF mechanism," in Proceedings of the 5th International IFIP-TC6 Networking Conference (NETWORKING '06), pp. 184-196, Coimbra, Portugal, May 2006.

[26] X. Tian, T. Sanada, T. Okuda, and T. Ideguchi, "A novel MAC protocol of wireless LAN with high throughput and fairness," in Proceedings of the 38th Annual IEEE Conference on Local Computer Networks (LCN '13), pp. 671-674, Sydney, Australia, October 2013.

[27] J.-S. Kim, E. Serpedin, and D.-R. Shin, "Improved particle filtering-based estimation of the number of competing stations in IEEE 802.11 networks," IEEE Signal Processing Letters, vol. 15, pp. 87-90, 2008.

[28] Y. Peng, H. Wu, S. Cheng, and K. Long, "A new self-adapt DCF algorithm," in Proceedings of the IEEE Global Telecommunications Conference (GLOBECOM '02), pp. 87-91, Taipei, Taiwan, November 2002.

[29] P. Starzetz, M. Heusse, F. Rousseau, and A. Duda, "Hashing backoff: a collision-free wireless access method," in NETWORKING 2009: 8th International IFIP-TC 6 Networking Conference, Aachen, Germany, May 11-15, 2009. Proceedings, vol. 5550 of Lecture Notes in Computer Science, pp. 11-15, Springer, Berlin, Germany, 2009. 
[30] J. Lee and I. Yeom, "Avoiding collision with hidden nodes in IEEE 802.11 wireless networks," IEEE Communications Letters, vol. 13, no. 10, pp. 743-745, 2009.

[31] S. Pudasaini, M. Kang, S. Shin, and J. A. Copeland, "COMIC: intelligent contention window control for distributed medium access," IEEE Communications Letters, vol. 14, no. 7, pp. 656$658,2010$.

[32] M. Heusse, F. Rousseau, R. Guillier, and A. Duda, "Idle sense: an optimal access method for high throughput and fairness in rate diverse wireless LANs," in Proceedings of the ACM Conference on Applications, Technologies, Architectures, and Protocols for Computer Communications (SIGCOMM '05), pp. 121-132, August 2005.

[33] S. Chun, D. Xianhua, L. Pingyuan, and Z. Han, "Adaptive access mechanism with optimal contention window based on node number estimation using multiple thresholds," IEEE Transactions on Wireless Communications, vol. 11, no. 6, pp. 2046-2055, 2012.

[34] C. Quan, M. H. Sook, and C. G. Young, "Estimation for the number of tags in the slotted-ALOHA based RFID systems," in Proceedings of the 6th International Conference on Computers, Communications and System, pp. 203-206, November 2005.

[35] G. B. Thomas, M. D. Weir, and J. Hass, Thomas' Calculus: Early Transcendentals, Addison-Wesley, New York, NY, USA, 12th edition, 2010.

[36] M. Adnan and E.-C. Park, "Hybrid control of contention window and frame aggregation for performance enhancement in multirate WLANs," Mobile Information Systems, vol. 2015, Article ID 383081, 16 pages, 2015.

[37] E. Ziouva and T. Antonakopoulos, "CSMA/CA performance under high traffic conditions: throughput and delay analysis," Computer Communications, vol. 25, no. 3, pp. 313-321, 2002. 

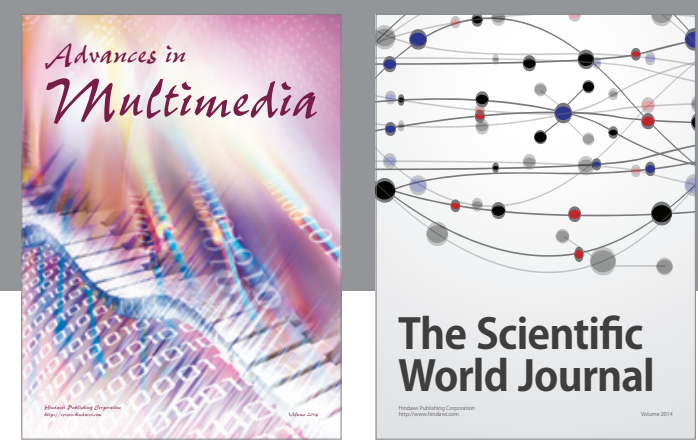

The Scientific World Journal
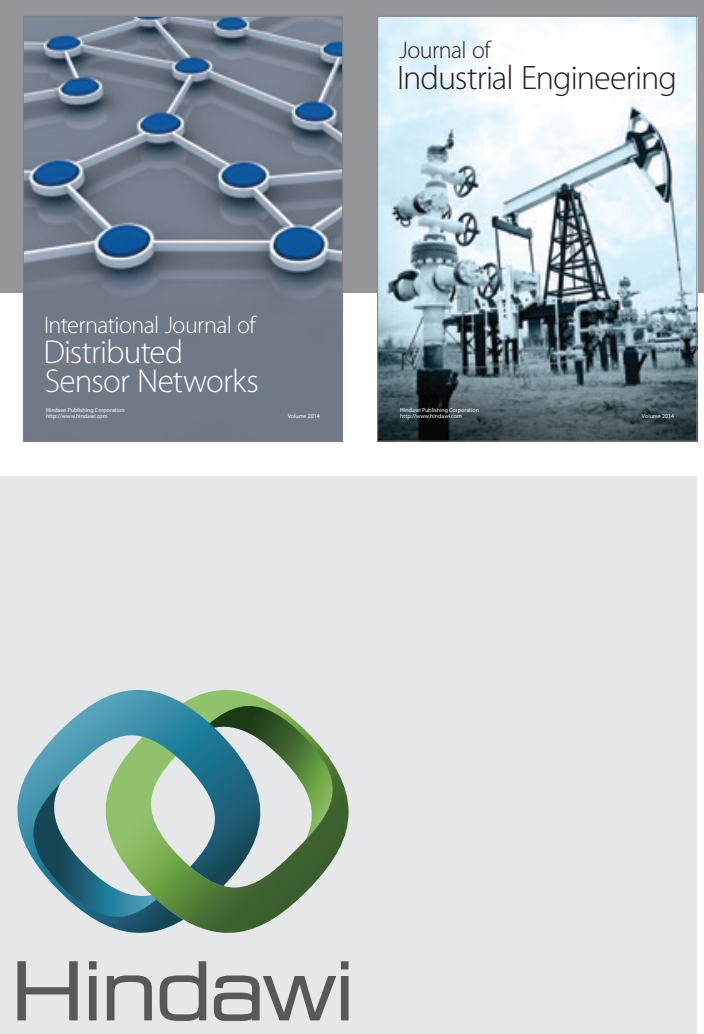

Submit your manuscripts at

http://www.hindawi.com

\section{Computer Networks} and Communications
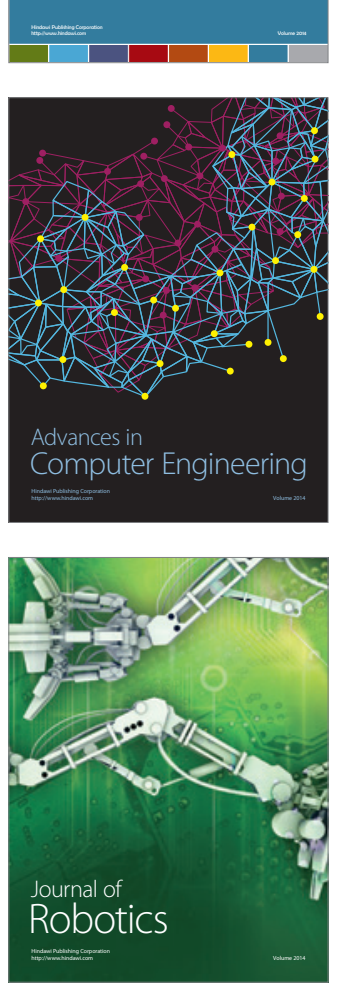
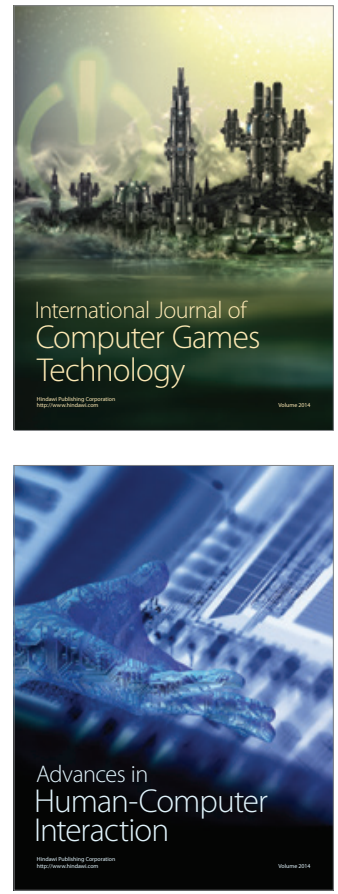
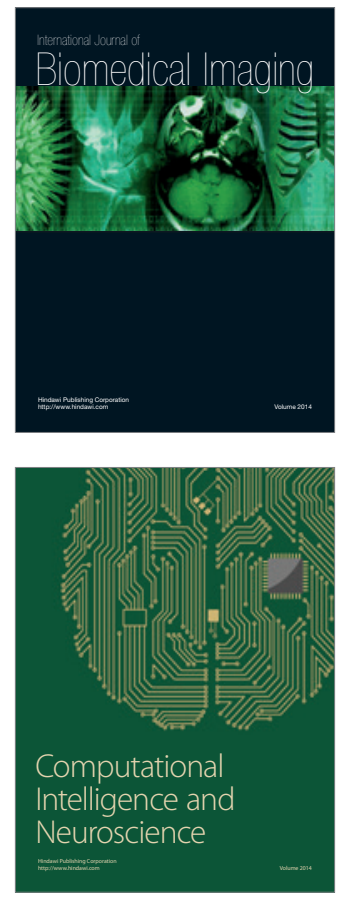
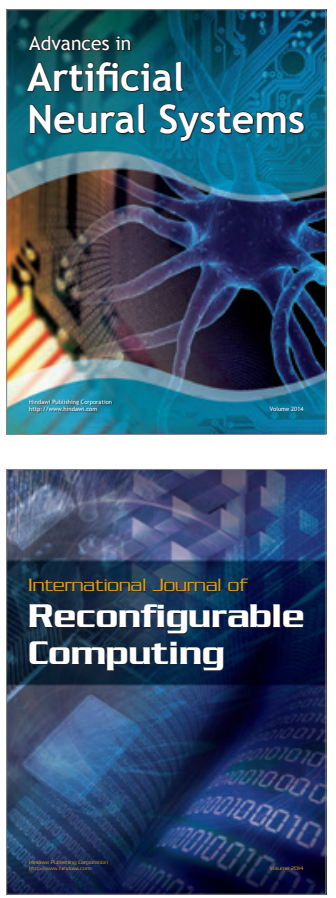
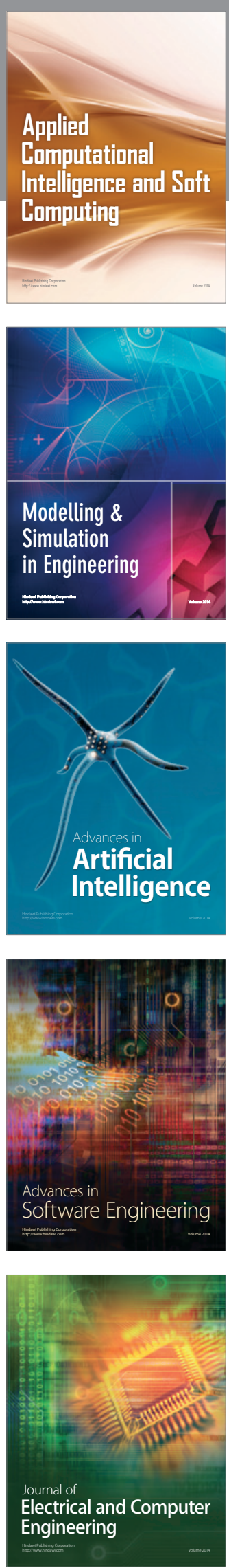